TGU-35

UWThPh-2005-13

HEPHY-PUB 811/05

IFIC/05-31

ZU-TH 10/05

\title{
Test of lepton flavour violation at LHC
}

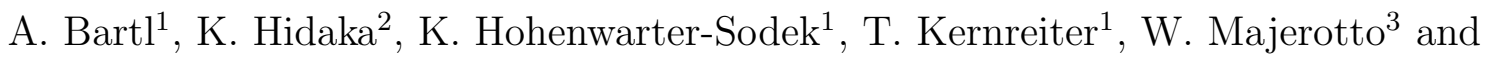 \\ W. $\operatorname{Porod}^{4,5}$ \\ 1 Institut für Theoretische Physik, Universität Wien, \\ A-1090 Vienna, Austria \\ 2 Department of Physics, Tokyo Gakugei University, Koganei, \\ Tokyo 184-8501, Japan \\ 3 Institut für Hochenergiephysik der Österreichischen Akademie der Wissenschaften, \\ A-1050, Austria \\ 4 Instituto de Física Corpuscular / C.S.I.C.- Universitat de Valencia, Edificio \\ Institutos de Paterna, Apartado de Correos 22085, E-46071 Valencia, Spain \\ 5 Institut für Theoretische Physik, Universität Zürich, \\ CH-8057 Zürich, Switzerland
}

\begin{abstract}
We study lepton flavour violating decays of neutralinos and sleptons within the Minimal Supersymmetric Standard Model, assuming two and three generation mixings in the slepton sector. We take into account the most recent bounds on flavour violating rare lepton decays. Taking the SPS1a' scenario as an example, we show that some of the lepton flavour violating branching ratios of neutralinos and sleptons can be sizable $(\sim 5-10 \%)$. We study the impact of the lepton flavour violating neutralino and slepton decays on the di-lepton mass distributions measured at LHC. We find that they can result in novel and characteristic edge structures in the distributions. In particular, double-edge structures can appear in the $e \tau$ and $\mu \tau$ mass spectra if $\tilde{\tau}_{1}$ is the lightest slepton. The appearance of these remarkable structures provides a powerful test of supersymmetric lepton flavour violation at LHC.
\end{abstract}




\section{Introduction}

There are stringent experimental constraints on lepton flavour violation (LFV) in the charged lepton sector, the strongest coming from the decay branching ratio of $\mu^{-} \rightarrow e^{-} \gamma, \operatorname{BR}\left(\mu^{-} \rightarrow e^{-} \gamma\right)<1.2 \times 10^{-11}$ [1]. Others are $\operatorname{BR}\left(\mu^{-} \rightarrow e^{-} e^{+} e^{-}\right)<10^{-12}$ [2], $\operatorname{BR}\left(\tau^{-} \rightarrow e^{-} \gamma\right)<1.1 \times 10^{-7}$ [3], $\operatorname{BR}\left(\tau^{-} \rightarrow \mu^{-} \gamma\right)<6.8 \times 10^{-8}$ [4], $\operatorname{BR}\left(\tau^{-} \rightarrow\right.$ $\left.\mu^{-} \mu^{+} \mu^{-}\right)<1.9 \times 10^{-7}$ [5] and the limit on $\mu^{-} N \rightarrow e^{-} N, R_{\mu e}<7.8 \times 10^{-13}$ [6], with $R_{\mu e}=\Gamma\left[\mu^{-} N(Z, A) \rightarrow e^{-} N(Z, A)\right] / \Gamma\left[\mu^{-} N(Z, A) \rightarrow \nu_{\mu} N(Z-1, A)\right]$. In particular, the bounds on $\operatorname{BR}\left(\tau^{-} \rightarrow e^{-} \gamma\right)$ and $\operatorname{BR}\left(\tau^{-} \rightarrow \mu^{-} \gamma\right)$ have recently been substantially improved.

On the other hand, the various neutrino experiments have clearly established that individual lepton flavour is violated (for a recent review see e.g. [7]). In supersymmetric (SUSY) extensions of the Standard Model LFV can originate in the slepton sector due to soft SUSY breaking parameters, e.g. mass matrices with flavour off-diagonal entries. Several studies along this line have been performed assuming either specific high-scale models or specifying the LFV parameters at the low scale (see for instance [8, 9, 10, 11]).

For the LHC it has been shown that SUSY LFV can be observed by studying the LFV decays of the second neutralino $\tilde{\chi}_{2}^{0}$ arising from cascade decays of gluinos and squarks, i.e. $\tilde{\chi}_{2}^{0} \rightarrow \tilde{\ell} \ell^{\prime} \rightarrow \ell^{\prime} \ell^{\prime \prime} \tilde{\chi}_{1}^{0}$ : signals of SUSY LFV can be extracted despite considerable backgrounds and stringent experimental bounds on flavour violating lepton decays in case of two generation mixings in either the right or left slepton sector in the mSUGRA model [12, 13, 14. The $\tilde{e}_{R}-\tilde{\mu}_{R}$ mixing case was studied in [12, 14, and the $\tilde{\mu}_{L}-\tilde{\tau}_{L}$ mixing case in [13].

In this paper we study the cases of two and three generation mixings in the slepton sector. We take the SPS1a' point as a reference scenario and work out in detail the individual branching ratios of the LFV two-body decays of the neutralino and sleptons. An interesting feature of this scenario is that the $\tilde{\tau}_{1}$ is lighter than the $\tilde{e}_{R}$ and $\tilde{\mu}_{R}$. As we will show, in the LFV case this leads to a novel double-edge structure in the $e \tau$ and $\mu \tau$ invariant mass distributions, not appearing in the cases previously studied.

We take into account the contraints on the LFV parameters from the most recent experimental limits on the rare decays $\ell^{-} \rightarrow \ell^{\prime-} \gamma$. This practically implies that the 
constraints from the rare three-body decays are fulfilled as they are dominated by virtual photon exchange [8]. The only possible exception is that from $\tau^{-} \rightarrow \mu^{-} \mu^{+} \mu^{-}$ enhanced by the Higgs boson exchange for large $\tan \beta$ [15]. This, however, does not apply to our case as we study a scenario with $\tan \beta=10$ in our numerical analysis. In the considered parameter range also the rate for $\mu-e$ conversion is well below the corresponding experimental limit [8].

\section{The model}

The most general charged slepton mass matrix including left-right mixing as well as flavour mixing in the basis of $\left(\tilde{e}_{L}, \tilde{\mu}_{L}, \tilde{\tau}_{L}, \tilde{e}_{R}, \tilde{\mu}_{R}, \tilde{\tau}_{R}\right) \equiv\left(\tilde{\ell}_{1 L}, \tilde{\ell}_{2 L}, \tilde{\ell}_{3 L}, \tilde{\ell}_{1 R}, \tilde{\ell}_{2 R}, \tilde{\ell}_{3 R}\right)$ is given by:

$$
M_{\tilde{\ell}}^{2}=\left(\begin{array}{cc}
M_{L L}^{2} & M_{R L}^{2 \dagger} \\
M_{R L}^{2} & M_{R R}^{2}
\end{array}\right),
$$

where the entries are $3 \times 3$ matrices. They are given by

$$
\begin{aligned}
M_{L L, \alpha \beta}^{2} & =M_{L, \alpha \beta}^{2}+\frac{v_{d}^{2} Y_{\alpha \gamma}^{E *} Y_{\beta \gamma}^{E}}{2}+\frac{\left(g^{\prime 2}-g^{2}\right)\left(v_{d}^{2}-v_{u}^{2}\right) \delta_{\alpha \beta}}{8}, \\
M_{R L, \alpha \beta}^{2} & =\frac{v_{d} A_{\beta \alpha}-\mu^{*} v_{u} Y_{\beta \alpha}^{E}}{\sqrt{2}}, \\
M_{R R, \alpha \beta}^{2} & =M_{E, \alpha \beta}^{2}+\frac{v_{d}^{2} Y_{\gamma \alpha}^{E} Y_{\gamma \beta}^{E *}}{2}-\frac{g^{\prime 2}\left(v_{d}^{2}-v_{u}^{2}\right) \delta_{\alpha \beta}}{4} .
\end{aligned}
$$

The indices $\alpha, \beta, \gamma=1,2,3$ characterize the flavours $e, \mu, \tau$, respectively. $M_{L}^{2}$ and $M_{E}^{2}$ are the hermitean soft SUSY breaking mass matrices for left and right sleptons, respectively. $A_{\alpha \beta}$ are the trilinear soft SUSY breaking couplings of the sleptons and the Higgs boson: $\mathcal{L}_{\text {int }}=-A_{\alpha \beta} \tilde{\ell}_{\beta R}^{\dagger} \tilde{\ell}_{\alpha L} H_{1}^{0}+\cdots \cdot v_{u}$ and $v_{d}$ are the vacuum expectation values of the Higgs fields with $v_{u}=\sqrt{2}\left\langle H_{2}^{0}\right\rangle, v_{d}=\sqrt{2}\left\langle H_{1}^{0}\right\rangle$, and $\tan \beta \equiv v_{u} / v_{d}$. We work in a basis where the Yukawa coupling matrix $Y_{\alpha \beta}^{E}$ of the charged leptons is real and flavour diagonal with $Y_{\alpha \alpha}^{E}=\sqrt{2} m_{\ell_{\alpha}} / v_{d}\left(\ell_{\alpha}=e, \mu, \tau\right)$. The physical mass eigenstates $\tilde{\ell}_{i}$ are given by $\tilde{\ell}_{i}=R_{i \alpha}^{\tilde{\ell}} \tilde{\ell}_{\alpha}^{\prime}$ with $\tilde{\ell}_{\alpha}^{\prime}=\left(\tilde{e}_{L}, \tilde{\mu}_{L}, \tilde{\tau}_{L}, \tilde{e}_{R}, \tilde{\mu}_{R}, \tilde{\tau}_{R}\right)$. The mixing matrix $R^{\tilde{\ell}}$ and the physical mass eigenvalues are obtained by an unitary transformation $R^{\tilde{\ell}} M_{\tilde{\ell}}^{2} R^{\tilde{\ell} \dagger}=\operatorname{diag}\left(m_{\tilde{\ell}_{1}}^{2}, \ldots, m_{\tilde{\ell}_{6}}^{2}\right)$, where $m_{\tilde{\ell}_{i}}<m_{\tilde{\ell}_{j}}$ for $i<j$. Similarly, one has for the 
sneutrinos in the basis of $\left(\tilde{\nu}_{e L}, \tilde{\nu}_{\mu L}, \tilde{\nu}_{\tau L}\right) \equiv\left(\tilde{\nu}_{e}, \tilde{\nu}_{\mu}, \tilde{\nu}_{\tau}\right)$

$$
M_{\tilde{\nu}, \alpha \beta}^{2}=M_{L, \alpha \beta}^{2}+\frac{\left(g^{2}+g^{\prime 2}\right)\left(v_{d}^{2}-v_{u}^{2}\right) \delta_{\alpha \beta}}{8} \quad(\alpha, \beta=1,2,3)
$$

with the physical mass eigenstates $\tilde{\nu}_{i}=R_{i \alpha}^{\tilde{\nu}} \tilde{\nu}_{\alpha}^{\prime}\left(m_{\tilde{\nu}_{1}}<m_{\tilde{\nu}_{2}}<m_{\tilde{\nu}_{3}}\right)$ and $\tilde{\nu}_{\alpha}^{\prime}=\left(\tilde{\nu}_{e}, \tilde{\nu}_{\mu}, \tilde{\nu}_{\tau}\right)$. The relevant interaction Lagrangian for this study in terms of mass eigenstates is given by:

$$
\begin{aligned}
\mathcal{L} & =\bar{\ell}_{i}\left(c_{i k m}^{L} P_{L}+c_{i k m}^{R} P_{R}\right) \tilde{\chi}_{k}^{0} \tilde{\ell}_{m}+\bar{\ell}_{i}\left(d_{i l j}^{L} P_{L}+d_{i l j}^{R} P_{R}\right) \tilde{\chi}_{l}^{-} \tilde{\nu}_{j}+\bar{\nu}_{i} e_{i k j}^{R} P_{R} \tilde{\chi}_{k}^{0} \tilde{\nu}_{j} \\
& +\bar{\nu}_{i} f_{i l m}^{R} P_{R} \tilde{\chi}_{l}^{+} \tilde{\ell}_{m}+\text { h.c. } .
\end{aligned}
$$

The specific forms of the couplings $c_{i k m}^{L}, c_{i k m}^{R}, d_{i l j}^{L}, d_{i l j}^{R}, e_{i k j}^{R}$ and $f_{i l m}^{R}$ can be found in [16]. The first two terms in Eq. (6) give rise to the LFV signals studied here, whereas the last one will give rise to the SUSY background because the neutrino flavour cannot be discriminated in high energy collider experiments.

\section{Lepton flavour violating decays of sleptons and neutralinos}

Now we discuss systematically LFV decays of charged sleptons and neutralinos. We will first consider cases where only two generations mix and afterwards the case of three generation mixing. For definitness, we consider the study point SPS1a' [17]. In this scenario we have a relatively light spectrum of charginos/neutralinos and sleptons with the three lighter charged sleptons being mainly $\tilde{\ell}_{R}$. This means that the flavour off-diagonal elements of $M_{E, \alpha \beta}^{2}$ in Eq. (4) are expected to give the most important contribution to the LFV decays of the lighter charginos/neutralinos and sleptons. We therefore discuss LFV only in the right slepton sector. For the SPS1a' point the relevant on-shell SUSY parameters are given by: $\tan \beta=10, M_{1}=100.1 \mathrm{GeV}, M_{2}=$ $197.4 \mathrm{GeV}, \mu=400 \mathrm{GeV} M_{L, 11}=M_{L, 22}=184 \mathrm{GeV}, M_{L, 33}=182.5 \mathrm{GeV}, M_{E, 11}=$ $117.793 \mathrm{GeV}, M_{E, 22}=117.797 \mathrm{GeV}, M_{E, 33}=111 \mathrm{GeV}, A_{11}=-0.013 \mathrm{GeV}, A_{22}=$ $-2.8 \mathrm{GeV}, A_{33}=-46 \mathrm{GeV}$. Here $M_{1}$ and $M_{2}$ are the $U(1)$ and $S U(2)$ gaugino mass parameters, respectively The mass spectrum of the lighter neutralinos and sleptons is: $m_{\tilde{\chi}_{1}^{0}}=97.8 \mathrm{GeV}, m_{\tilde{\chi}_{2}^{0}}=184 \mathrm{GeV}, m_{\tilde{e}_{1}}=125.251 \mathrm{GeV}, m_{\tilde{\mu}_{1}}=125.212 \mathrm{GeV}$, 
$m_{\tilde{\tau}_{1}}=107.4 \mathrm{GeV}$. We have taken $M_{E, 22}^{2}=M_{E, 11}^{2}+1 \mathrm{GeV}^{2}$, i.e. $M_{E, 22}=M_{E, 11}+4 \mathrm{MeV}$, to avoid potential numerical problems in the definition of the LFV mixing angles (see Eq. (9) below). This results in a mass difference $m_{\tilde{e}_{1}}-m_{\tilde{\mu}_{1}}=39 \mathrm{MeV}$ which is well below the expected mass resolution of LHC or ILC. We consider the following $\tilde{\chi}_{2}^{0}$ decays

$$
\tilde{\chi}_{2}^{0} \longrightarrow \ell_{i}^{ \pm} \tilde{\ell}_{j}^{\mp}
$$

where $\tilde{\ell}_{1}=\tilde{\tau}_{1} \sim \tilde{\tau}_{R}, \tilde{\ell}_{2}=\tilde{\mu}_{1} \simeq \tilde{\mu}_{R}$ and $\tilde{\ell}_{3}=\tilde{e}_{1} \simeq \tilde{e}_{R}$. They decay further as

$$
\tilde{\ell}_{j}^{\mp} \longrightarrow \ell_{k}^{\mp} \tilde{\chi}_{1}^{0}
$$

see Table [1.

As the next step in our analysis, we add lepton flavour violating real parameters $M_{E, \alpha \beta}^{2}$ with $\alpha \neq \beta$ inducing LFV decays of neutralinos/charginos and sleptons. It is convenient to define the following effective lepton flavour mixing angles

$$
\tan 2 \theta_{\alpha \beta}^{e f f} \equiv \frac{2 M_{E, \alpha \beta}^{2}}{M_{E, \alpha \alpha}^{2}-M_{E, \beta \beta}^{2}}, \quad(\alpha<\beta)
$$

which are a measure of LFV.

In Fig. 11 we show the lepton flavour violating branching ratios $\operatorname{BR}\left(\tilde{\ell}_{3}^{-} \rightarrow \mu^{-} \tilde{\chi}_{1}^{0}\right)$ and $\operatorname{BR}\left(\tilde{\chi}_{2}^{0} \rightarrow \tilde{\ell}_{3} \ell_{i}\right)$ as a function of $\tan 2 \theta_{12}^{e f f}$. The parameter $M_{E, 12}^{2}$ has been varied in the full range satisfying $\operatorname{BR}\left(\mu^{-} \rightarrow e^{-} \gamma\right)<1.2 \times 10^{-11}$, with $M_{E, 13}^{2}=M_{E, 23}^{2}=0$. Note that $\tilde{\ell}_{3}=\tilde{e}_{1} \simeq \tilde{e}_{R}$ for $\tan 2 \theta_{12}^{e f f}=0$ whereas $\tilde{\ell}_{3}$ is mainly $\tilde{e}_{1}$ with an admixture of $\tilde{\mu}_{R}$ for $\tan 2 \theta_{12}^{e f f} \neq 0$. As can be seen in Fig. 1(a) $\operatorname{BR}\left(\tilde{\ell}_{3}^{-} \rightarrow \mu^{-} \tilde{\chi}_{1}^{0}\right)$ can go up to $\sim 15 \%$. As $\tilde{\ell}_{2} \sim \tilde{\mu}_{1}$ and $\tilde{\ell}_{3} \sim \tilde{e}_{1}$, one has $\operatorname{BR}\left(\tilde{\ell}_{3} \rightarrow \mu \tilde{\chi}_{1}^{0}\right) \simeq \operatorname{BR}\left(\tilde{\ell}_{2} \rightarrow e \tilde{\chi}_{1}^{0}\right)$ for a fixed value of $\tan 2 \theta_{12}^{e f f}$. In Fig. 1(b) we show the LFV branching ratio $\operatorname{BR}\left(\tilde{\chi}_{2}^{0} \rightarrow \tilde{\ell}_{3} \mu\right)$ (full line); the $\mathrm{LFV}$ branching ratio $\operatorname{BR}\left(\tilde{\chi}_{2}^{0} \rightarrow \tilde{\ell}_{2} e\right)$ has the same value. We also show the "lepton flavour conserving (LFC)" branching ratio $\mathrm{BR}\left(\tilde{\chi}_{2}^{0} \rightarrow \tilde{\ell}_{3} e\right)$ (dashed line); the branching ratio $\operatorname{BR}\left(\tilde{\chi}_{2}^{0} \rightarrow \tilde{\ell}_{2} \mu\right)$ again has the same value. The sum of the LFV branching ratios of $\tilde{\chi}_{2}^{0}, \operatorname{BR}\left(\tilde{\chi}_{2}^{0} \rightarrow \tilde{\ell}_{3} \mu\right)$ and $\operatorname{BR}\left(\tilde{\chi}_{2}^{0} \rightarrow \tilde{\ell}_{2} e\right)$, can reach about $0.6 \%$, which is about $1 / 6$ of the sum of "LFC" branching ratios $\operatorname{BR}\left(\tilde{\chi}_{2}^{0} \rightarrow \tilde{\ell}_{3} e\right)+\operatorname{BR}\left(\tilde{\chi}_{2}^{0} \rightarrow \tilde{\ell}_{2} \mu\right)$. Note that the dominant decay channels of $\tilde{\chi}_{2}^{0}$ are into $\tilde{\tau}_{1} \tau$ and $\tilde{\nu}_{\ell} \nu_{\ell}$ (see Table 1).

In Fig. 2 we take $M_{E, 13}^{2} \neq 0$ varying it in the full range satisfying $\operatorname{BR}\left(\tau^{-} \rightarrow e^{-} \gamma\right)<$ $1.1 \times 10^{-7}$, with $M_{E, 12}^{2}=M_{E, 23}^{2}=0$. In Fig. 2(a) we show the LFV branching ratio 
(a) $\operatorname{BR}\left(\tilde{\ell}_{3}^{-} \rightarrow \mu^{-} \tilde{\chi}_{1}^{0}\right)$

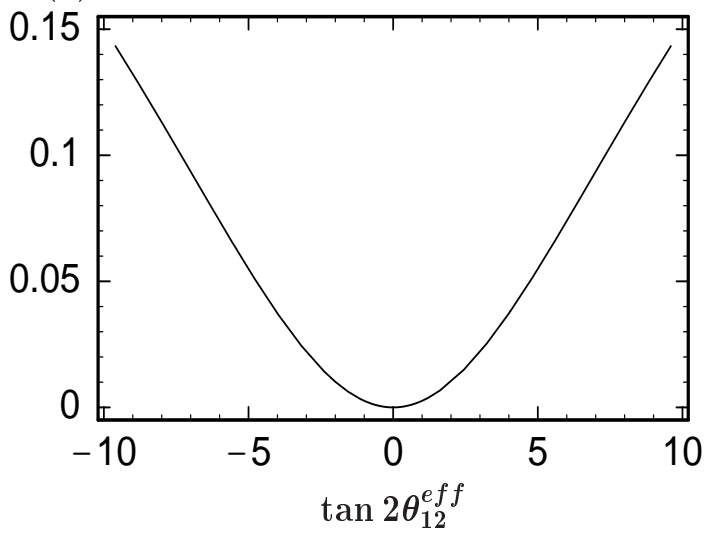

(b) $\operatorname{BR}\left(\tilde{\chi}_{2}^{0} \rightarrow \tilde{\ell}_{3} \ell_{i}\right)$

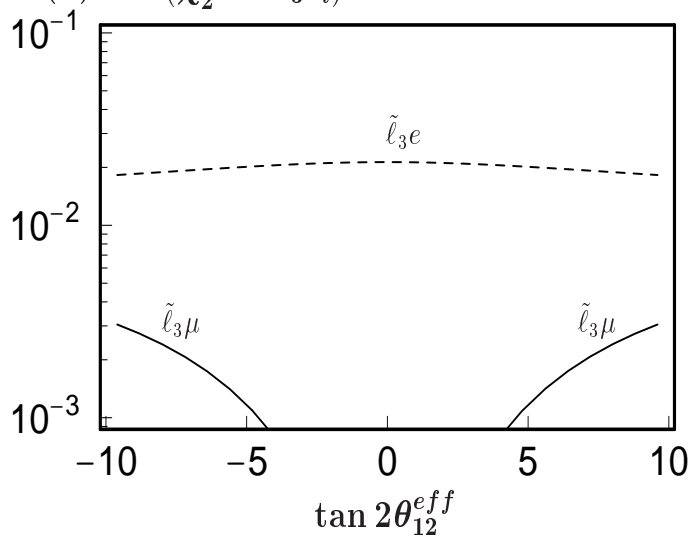

Figure 1: In (a) we show $\operatorname{BR}\left(\tilde{\ell}_{3}^{-} \rightarrow \mu^{-} \tilde{\chi}_{1}^{0}\right)$ as a function of $\tan 2 \theta_{12}^{\text {eff }}$ and in (b) $\operatorname{BR}\left(\tilde{\chi}_{2}^{0} \rightarrow \tilde{\ell}_{3} \mu\right)$ and $\operatorname{BR}\left(\tilde{\chi}_{2}^{0} \rightarrow \tilde{\ell}_{3} e\right)$ as a function of tan $2 \theta_{12}^{\text {eff }}$ summing over the charges, where $\tilde{\ell}_{3}$ is dominantly $\tilde{e}_{1}$ with an admixture of $\tilde{\mu}_{R}$.

$\operatorname{BR}\left(\tilde{\ell}_{1}^{-} \rightarrow e^{-} \tilde{\chi}_{1}^{0}\right)$ as a function of $\tan 2 \theta_{13}^{e f f}$, where $\tilde{\ell}_{1}\left(\tilde{\ell}_{3}\right)$ is dominantly $\tilde{\tau}_{1}\left(\tilde{e}_{1}\right)$ with an admixture of $\tilde{e}_{R}\left(\tilde{\tau}_{R}\right)$. The LFV branching ratio can go up to 3.5\%. In Fig. 2(b) we plot the LFV branching ratios $\operatorname{BR}\left(\tilde{\chi}_{2}^{0} \rightarrow \tilde{\ell}_{3} \tau\right)$ and $\operatorname{BR}\left(\tilde{\chi}_{2}^{0} \rightarrow \tilde{\ell}_{1} e\right)$ as well as the "LFC" branching ratios $\operatorname{BR}\left(\tilde{\chi}_{2}^{0} \rightarrow \tilde{\ell}_{1} \tau\right)$ and $\operatorname{BR}\left(\tilde{\chi}_{2}^{0} \rightarrow \tilde{\ell}_{3} e\right)$. The LFV branching ratio $\operatorname{BR}\left(\tilde{\chi}_{2} \rightarrow \tilde{\ell}_{3} \tau\right)$ can reach about $2 \%$. The relative magnitudes of the branching ratios in Fig. 2(b) are explained as follows: for the SPS1a' scenario we have $\tilde{\chi}_{2}^{0} \sim \tilde{W}^{3}$ so that the $\tilde{\chi}_{2}^{0}$ couples strongly to $\tilde{\ell}_{L}$ and only weakly to $\tilde{\ell}_{R}$. However, only in the $\tilde{\tau}$-sector there is a significant $\tilde{\ell}_{L}-\tilde{\ell}_{R}$ mixing. Therefore, for large tan $2 \theta_{13}^{e f f}$, the LFV branching ratio $\operatorname{BR}\left(\tilde{\chi}_{2}^{0} \rightarrow \tilde{\ell}_{3} \tau\right)$ has about the same size as the "LFC" branching ratio $\operatorname{BR}\left(\tilde{\chi}_{2}^{0} \rightarrow \tilde{\ell}_{3} e\right)$.

We have also considered the case $M_{E, 23}^{2} \neq 0, M_{E, 12}^{2}=M_{E, 13}^{2}=0$ for the LFV decays $\tilde{\ell}_{1}^{-} \rightarrow \mu^{-} \tilde{\chi}_{1}^{0}, \tilde{\chi}_{2}^{0} \rightarrow \tilde{\ell}_{2} \tau$ and $\tilde{\chi}_{2}^{0} \rightarrow \tilde{\ell}_{1} \mu\left(\tilde{\ell}_{1} \sim \tilde{\tau}_{1}\right.$ and $\left.\tilde{\ell}_{2} \sim \tilde{\mu}_{1}\right)$. In this case we have found similar behaviours as in the above case $\left(M_{E, 13}^{2} \neq 0, M_{E, 12}^{2}=M_{E, 23}^{2}=0\right)$ because the experimental bounds on $\operatorname{BR}\left(\tau^{-} \rightarrow e^{-} \gamma\right)$ and $\operatorname{BR}\left(\tau^{-} \rightarrow \mu^{-} \gamma\right)$ are similar.

In Fig. 3(a) we show $\operatorname{BR}\left(\tilde{\chi}_{2}^{0} \rightarrow e \tau \tilde{\chi}_{1}^{0}\right)$ summed over all intermediate sleptons and all charges as a function of $\operatorname{BR}\left(\tau^{-} \rightarrow e^{-} \gamma\right)$. The former branching ratio has been calculated by using the formula

$$
\begin{aligned}
\operatorname{BR}\left(\tilde{\chi}_{2}^{0} \rightarrow e \tau \tilde{\chi}_{1}^{0}\right)= & \sum_{i=1}^{3}\left[\operatorname{BR}\left(\tilde{\chi}_{2}^{0} \rightarrow e \tilde{\ell}_{i}\right) \operatorname{BR}\left(\tilde{\ell}_{i} \rightarrow \tau \tilde{\chi}_{1}^{0}\right)\right. \\
& \left.+\operatorname{BR}\left(\tilde{\chi}_{2}^{0} \rightarrow \tau \tilde{\ell}_{i}\right) \operatorname{BR}\left(\tilde{\ell}_{i} \rightarrow e \tilde{\chi}_{1}^{0}\right)\right] .
\end{aligned}
$$



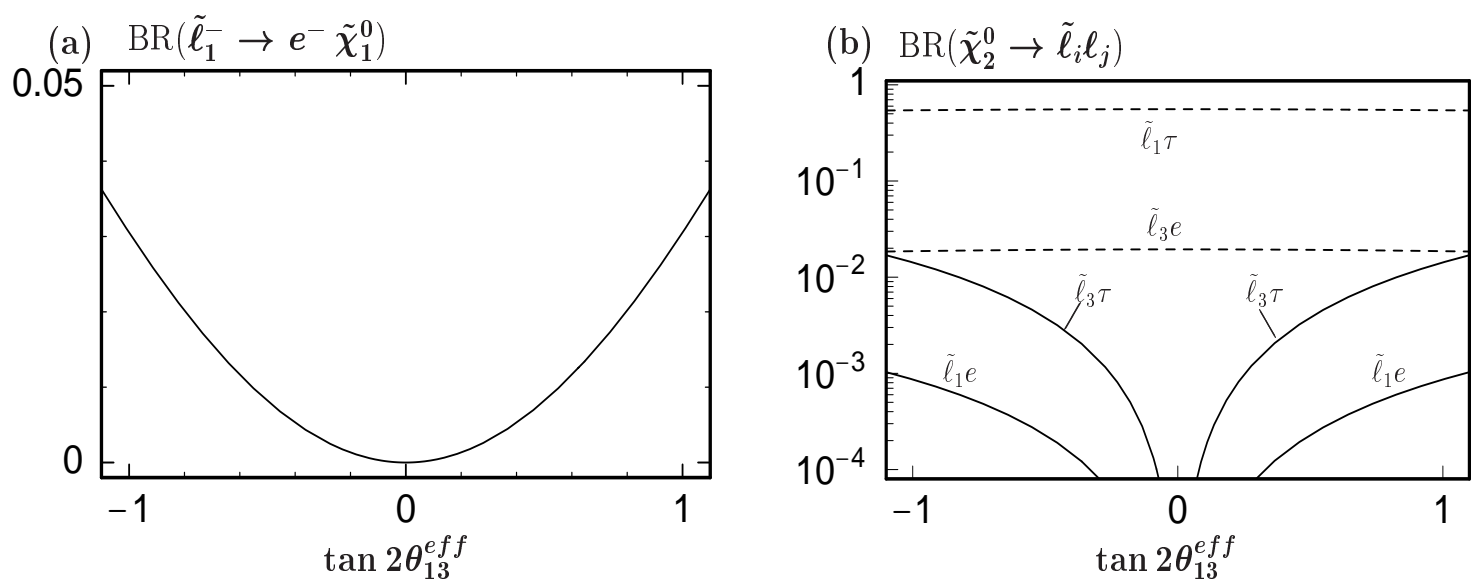

Figure 2: $\quad$ In (a) we show $\operatorname{BR}\left(\tilde{\ell}_{1}^{-} \rightarrow e^{-} \tilde{\chi}_{1}^{0}\right)$ as a function of $\tan 2 \theta_{13}^{\text {eff }}$ and in (b) $\operatorname{BR}\left(\tilde{\chi}_{2}^{0} \rightarrow \tilde{\ell}_{3} \tau\right), \operatorname{BR}\left(\tilde{\chi}_{2}^{0} \rightarrow \tilde{\ell}_{1} e\right), \operatorname{BR}\left(\tilde{\chi}_{2}^{0} \rightarrow \tilde{\ell}_{1} \tau\right)$ and $\operatorname{BR}\left(\tilde{\chi}_{2}^{0} \rightarrow \tilde{\ell}_{3} e\right)$ as a function of $\tan 2 \theta_{13}^{e f f}$ summing over the charges, where $\tilde{\ell}_{1}\left(\tilde{\ell}_{3}\right)$ is dominantly $\tilde{\tau}_{1}\left(\tilde{e}_{1}\right)$ with an admixture of $\tilde{e}_{R}\left(\tilde{\tau}_{R}\right)$.

We have randomly varied all off-diagonal entries in $M_{E}^{2}$ such that all experimental constraints due to the rare lepton decays are fulfilled at the same time. We see a strong correlation between $\operatorname{BR}\left(\tilde{\chi}_{2}^{0} \rightarrow e \tau \tilde{\chi}_{1}^{0}\right)$ and $\operatorname{BR}\left(\tau^{-} \rightarrow e^{-} \gamma\right)$. This correlation appears since both $\operatorname{BR}\left(\tilde{\chi}_{2}^{0} \rightarrow e \tau \tilde{\chi}_{1}^{0}\right)$ and $\operatorname{BR}\left(\tau^{-} \rightarrow e^{-} \gamma\right)$ depend strongly on the parameter $M_{E, 13}$. We have found a similar strong correlation between $\operatorname{BR}\left(\tilde{\chi}_{2}^{0} \rightarrow \mu \tau \tilde{\chi}_{1}^{0}\right)$ and $\operatorname{BR}\left(\tau^{-} \rightarrow \mu^{-} \gamma\right)$. In Fig. 通(b) we show the branching ratio $\operatorname{BR}\left(\tilde{\chi}_{2}^{0} \rightarrow e \mu \tilde{\chi}_{1}^{0}\right)$ and find its upper bound of about $2 \%$ almost independent of $\operatorname{BR}\left(\mu^{-} \rightarrow e^{-} \gamma\right)$. This independence can be understood in the following way: In the mass insertion approximation, there are two contributions to $\mu^{-} \rightarrow e^{-} \gamma$. The first one is due to $\tilde{\mu}-\tilde{e}$ mixing and the second one is due to the product of $\tilde{\mu}-\tilde{\tau}$ and $\tilde{\tau}-\tilde{e}$ mixings. As the constraints on rare $\tau$ decays are much less stringent than those on rare $\mu$ decays, the second one can be destructive and as important as the first one. This means that sizable $\operatorname{BR}\left(\tilde{\chi}_{2}^{0} \rightarrow e \mu \tilde{\chi}_{1}^{0}\right)$ is possible even for smaller $\operatorname{BR}\left(\mu^{-} \rightarrow e^{-} \gamma\right)$ in case of three generation mixing differently from that of two generation mixing as studied in [12, 13, 14] ${ }^{1}$. Note that, in the SPS1a' scenario the masses of $\tilde{\ell}_{2}$ and $\tilde{\ell}_{3}\left(\tilde{\ell}_{2} \simeq \tilde{\mu}_{R}, \tilde{\ell}_{3} \simeq \tilde{e}_{R}\right)$ are nearly degenerate and, hence, interference terms due to slepton flavour oscillation may reduce $\operatorname{BR}\left(\tilde{\chi}_{2}^{0} \rightarrow\right.$ e $\left.\mu \tilde{\chi}_{1}^{0}\right)$ significantly [11]. We have found numerically that the formula in Eq. (10) with $\tau$

\footnotetext{
${ }^{1}$ Another possibility to enhance $\operatorname{BR}\left(\tilde{\chi}_{2}^{0} \rightarrow e \mu \tilde{\chi}_{1}^{0}\right)$ is to choose certain ratios between the higgsino mass parameter $\mu$ and the gaugino mass parameter $M_{2}$ [14.
} 
(a) $\operatorname{BR}\left(\tilde{\chi}_{2}^{0} \rightarrow e \tau \tilde{\chi}_{1}^{0}\right)$

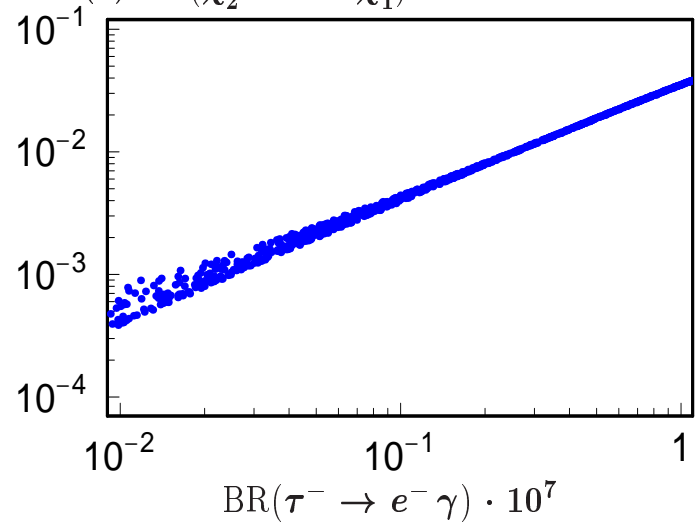

(b) $\operatorname{BR}\left(\tilde{\chi}_{2}^{0} \rightarrow e \mu \tilde{\chi}_{1}^{0}\right)$

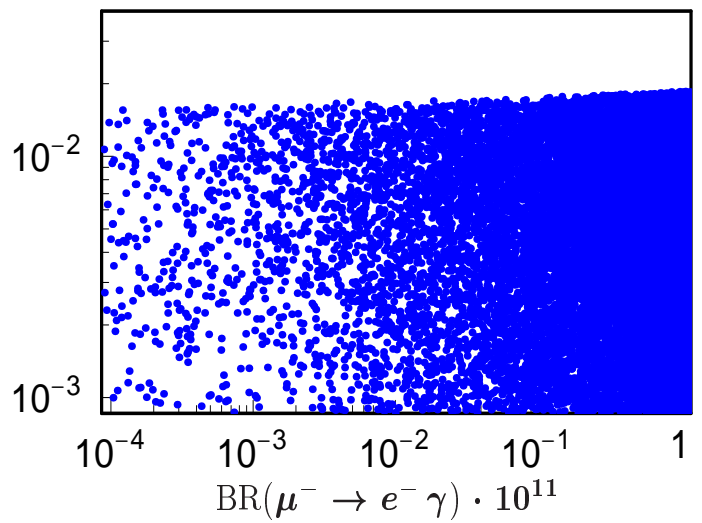

Figure 3: LFV decay branching ratios $\operatorname{BR}\left(\tilde{\chi}_{2}^{0} \rightarrow e \tau \tilde{\chi}_{1}^{0}\right)$ and $\operatorname{BR}\left(\tilde{\chi}_{2}^{0} \rightarrow e \mu \tilde{\chi}_{1}^{0}\right)$ as a function of $\operatorname{BR}\left(\tau^{-} \rightarrow e^{-} \gamma\right)$ and $\operatorname{BR}\left(\mu^{-} \rightarrow e^{-} \gamma\right)$, respectively, varying the LFV parameters around the SPS1a' point.

replaced by $\mu$ reproduces the correct results within $10 \%$ error if $\left(m_{\tilde{\ell}_{3}}-m_{\tilde{\ell}_{2}}\right) / \Gamma \geq 3$ $\left[\Gamma=\Gamma_{\tilde{\ell}_{2}} \simeq \Gamma_{\tilde{\ell}_{3}}\right]$.

\section{Effects on di-lepton invariant mass spectra}

Now we consider LFV effects on the di-lepton mass distribution in $\tilde{\chi}_{2}^{0}$ decays

$$
\tilde{\chi}_{2}^{0} \rightarrow \tilde{\ell}_{i}^{+} \ell_{j}^{-} \rightarrow \ell_{k}^{+} \ell_{j}^{-} \tilde{\chi}_{1}^{0}
$$

These decays can appear in the cascade decays of squarks and gluinos as produced at LHC. In these events one studies the invariant di-lepton mass spectrum $d N / d m(\ell \ell)$ with $m(\ell \ell)^{2}=\left(p_{\ell^{+}}+p_{\ell^{-}}\right)^{2}$. Its kinematical endpoint is used in combination with other observables to determine masses or mass differences of sparticles [18, 19, 20]. These spectra will change in the presence of lepton flavour violation.

To illustrate the effect of LFV on these spectra, in Fig. 4 we present invariant mass distributions for various lepton pairs taking the following LFV parameters: $M_{E, 12}^{2}=30 \mathrm{GeV}^{2}, M_{E, 13}^{2}=850 \mathrm{GeV}^{2}$ and $M_{E, 23}^{2}=600 \mathrm{GeV}^{2}$, for which we have $\left(m_{\tilde{\ell}_{1}}, m_{\tilde{\ell}_{2}}, m_{\tilde{\ell}_{3}}\right)=(106.4,125.1,126.2) \mathrm{GeV}$. These parameters are chosen such that large LFV $\tilde{\chi}_{2}^{0}$ decay branching ratios are possible consistently with the experimental bounds on the rare lepton decays (see Fig. 3). For this set of pa- 
rameters we obtain $\operatorname{BR}\left(\mu^{-} \rightarrow e^{-} \gamma\right)=9.5 \times 10^{-12}, \operatorname{BR}\left(\tau^{-} \rightarrow e^{-} \gamma\right)=1.0 \times 10^{-7}$ and $\operatorname{BR}\left(\tau^{-} \rightarrow \mu^{-} \gamma\right)=5.2 \times 10^{-8}$. In Table 1 we show the slepton and $\tilde{\chi}_{2}^{0}$ decay branching ratios in the LFV case (as well as in the lepton flavour conserving (LFC) case). By using Table 1 and the formulae analogous to Eq. (101) we obtain the following $\tilde{\chi}_{2}^{0}$ decay branching ratios in this $\operatorname{LFV}$ case: $\operatorname{BR}(e \mu)=1.7 \%, \operatorname{BR}(e \tau)=3.4 \%$, $\mathrm{BR}(\mu \tau)=1.8 \%, \operatorname{BR}\left(e^{+} e^{-}\right)=1 \%, \operatorname{BR}\left(\mu^{+} \mu^{-}\right)=1.2 \%, \operatorname{BR}\left(\tau^{+} \tau^{-}\right)=51 \%$ with $\operatorname{BR}\left(\ell_{i} \ell_{j}\right) \equiv \operatorname{BR}\left(\tilde{\chi}_{2}^{0} \rightarrow \ell_{i} \ell_{j} \tilde{\chi}_{1}^{0}\right)$. In Fig. 4(a) we show the flavour violating spectra $\left(100 / \Gamma_{t o t}\right) d \Gamma\left(\tilde{\chi}_{2}^{0} \rightarrow \ell_{i}^{ \pm} \ell_{j}^{\mp} \tilde{\chi}_{1}^{0}\right) / d m\left(\ell_{i}^{ \pm} \ell_{j}^{\mp}\right)$ versus $m\left(\ell_{i}^{ \pm} \ell_{j}^{\mp}\right)$ for the final states $\mu \tau$, e $\tau$ and $e \mu$. In cases where the final state contains a $\tau$-lepton, one finds two sharp edges. The first one at $m \simeq 59.4 \mathrm{GeV}$ is due to an intermediate $\tilde{\ell}_{1}\left(\sim \tilde{\tau}_{R}\right)$ and the second one at $m \simeq 84.6 \mathrm{GeV}$ is due to intermediate states of the two heavier sleptons $\tilde{\ell}_{2}\left(\sim \tilde{\mu}_{R}\right)$ and $\tilde{\ell}_{3}\left(\sim \tilde{e}_{R}\right)$ with $m_{\tilde{\ell}_{2}} \simeq m_{\tilde{\ell}_{3}}$ (see Eq. (10) $)$. The position of the edges can be expressed in terms of the neutralino and intermediate slepton masses [18]:

$$
m_{\text {edge }}^{2}(\ell \ell)=\frac{\left(m_{\tilde{\chi}_{2}^{0}}^{2}-m_{\tilde{\ell}_{i}}^{2}\right)\left(m_{\tilde{\ell}_{i}}^{2}-m_{\tilde{\chi}_{1}^{0}}^{2}\right)}{m_{\tilde{\ell}_{i}}^{2}}
$$

In the case of the $e \mu$ spectrum the first edge is practically invisible because the branching ratios of $\tilde{\chi}_{2}^{0}$ into $\tilde{\ell}_{1} e$ and $\tilde{\ell}_{1} \mu$ are tiny as can be seen in Table 1 Note that the rate for the $e \tau$ final state is largest in our case because $\left|M_{E, 13}^{2}\right|$ is larger than the other LFV parameters.

In Fig. 4(b) we show the "flavour conserving" spectra for the final states with $e^{+} e^{-}$and $\mu^{+} \mu^{-}$. The dashed line corresponds to the flavour conserving case where $M_{E, i j}^{2}=0$ for $i \neq j$. LFV causes firstly a reduction of the height of the end point peak. Secondly, it induces a difference between the $\mu^{+} \mu^{-}$and $e^{+} e^{-}$spectra because the mixings among the three slepton generations are in general different from one another. The peaks at $m \simeq 59.4 \mathrm{GeV}$ in the $\mu^{+} \mu^{-}$and $e^{+} e^{-}$spectra are invisible as in the $e \mu$ spectrum, for the same reason as mentioned above. As for the $\tau^{+} \tau^{-}$spetrum we remark that the height of the peak (due to the intermediate $\tilde{\ell}_{1}\left(\sim \tilde{\tau}_{R}\right)$ ) in the $\tau^{+} \tau^{-}$ spetrum gets reduced by about $5 \%$ and that the contributions due to the intermediate $\tilde{\ell}_{2,3}$ are invisible. Moreover, the peak position gets shifted to a smaller value by about $2.7 \mathrm{GeV}$ since the mass of the intermediate $\tilde{\ell}_{1}$ gets reduced by $1 \mathrm{GeV}$ compared to the flavour conserving case.

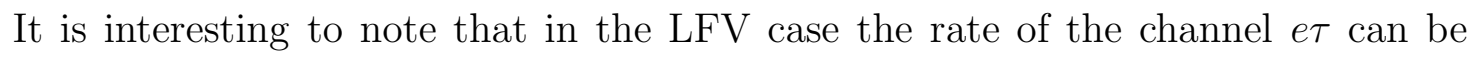


larger than those of the channels with the same flavour, $e^{+} e^{-}$and $\mu^{+} \mu^{-}$. Moreover, by measuring all di-lepton spectra for the flavour violating as well as conserving channels, one can make an important cross check of this LFV scenario: the first peak position of the lepton flavour violating spectra (except the $e \mu$ spectrum) must coincide with the end point of the $\tau^{+} \tau^{-}$spectrum and the second peak must coincide with those of the $e^{+} e^{-}$and $\mu^{+} \mu^{-}$spectra.

Up to now we have investigated in detail the di-lepton mass spectra taking SPS1a' as a specific example. In the following we discuss which requirements other scenarios must fulfill to observe double-edge structures. Obviously the kinematic condition $m_{\tilde{\chi}_{s}^{0}}>m_{\tilde{\ell}_{i}, \tilde{\ell}_{j}}>m_{\tilde{\chi}_{r}^{0}}$ must be fulfilled and sufficiently many $\tilde{\chi}_{s}^{0}$ must be produced. In addition there should be two sleptons contributing in a sizable way to the decay $\tilde{\chi}_{s}^{0} \rightarrow \ell^{\prime} \ell^{\prime \prime} \tilde{\chi}_{r}^{0}$ and, of course, the corresponding branching ratio has to be large enough to be observed. For this the corresponding LFV entries in the slepton mass matrix have to be large enough. Moreover, also the mass difference between the two contributing sleptons has to be sufficiently large so that the difference of the positions of the two peaks is larger than the experimental resolution. In mSugra-like scenarios the kinematic requirements (including the positions of the peaks) are fulfilled in the regions of parameter space where $m_{0}^{2} \lesssim 0.4 m_{1 / 2}^{2}$ and $\tan \beta \gtrsim 8$. The first condition provides for right sleptons lighter than the $\tilde{\chi}_{2}^{0}$ and the second condition ensures that the mass difference between $\tilde{\tau}_{1}$ and the other two right sleptons is sufficiently large. In the region where $m_{0}^{2} \lesssim 0.05 m_{1 / 2}^{2}$ also the left sleptons are lighter than $\tilde{\chi}_{2}^{0}$, giving the possibility of additional structures in the di-lepton mass spectra. We remark that SPS1a' is not the most favourable case because of the appearance of the decay $\tilde{\chi}_{2}^{0} \rightarrow \nu \nu \tilde{\chi}_{1}^{0}$ which has quite a large branching ratio $(\sim 40 \%)$. For example in the original SPS1a point this decay mode is absent allowing for much larger LFV branching ratios of $\tilde{\chi}_{2}^{0}$.

Finally we briefly discuss background reactions in the LFV search at LHC. The largest SM background is due to $t \bar{t}$ production. There is also SUSY background due to uncorrelated leptons stemming from different squark and gluino decay chains. The resulting di-lepton mass distributions will, however, be smooth and decrease monotonically with increasing di-lepton invariant mass as was explicitly shown in a Monte Carlo analysis in 13, 14. It was also shown that the single edge structure can be observed over the smooth background in the $e \mu$ and $\mu \tau$ invariant mass distributions. Therefore the novel distributions as shown in Fig. 4, in particular the characteristic double-edge 


\begin{tabular}{|c|c|c||c|c|c|}
\hline channel & LFC case & LFV case & channel & LFC case & LFV case \\
\hline$\tilde{\ell}_{1} \rightarrow \tilde{\chi}_{1}^{0} e$ & 0 & 0.034 & $\tilde{\chi}_{2}^{0} \rightarrow \tilde{\ell}_{1}^{ \pm} e^{\mp}$ & 0 & 0.001 \\
$\tilde{\ell}_{1} \rightarrow \tilde{\chi}_{1}^{0} \mu$ & 0 & 0.017 & $\tilde{\chi}_{2}^{0} \rightarrow \tilde{\ell}_{1}^{ \pm} \mu^{\mp}$ & 0 & 0.0005 \\
$\tilde{\ell}_{1} \rightarrow \tilde{\chi}_{1}^{0} \tau$ & 1 & 0.949 & $\tilde{\chi}_{2}^{0} \rightarrow \tilde{\ell}_{1}^{ \pm} \tau^{\mp}$ & 0.558 & 0.535 \\
\cline { 1 - 2 }$\tilde{\ell}_{2} \rightarrow \tilde{\chi}_{1}^{0} e$ & 0 & 0.35 & $\tilde{\chi}_{2}^{0} \rightarrow \tilde{\ell}_{2}^{ \pm} e^{\mp}$ & 0 & 0.007 \\
$\tilde{\ell}_{2} \rightarrow \tilde{\chi}_{1}^{0} \mu$ & 1 & 0.649 & $\tilde{\chi}_{2}^{0} \rightarrow \tilde{\ell}_{2}^{ \pm} \mu^{\mp}$ & 0.021 & 0.014 \\
$\tilde{\ell}_{2} \rightarrow \tilde{\chi}_{1}^{0} \tau$ & 0 & 0.00002 & $\tilde{\chi}_{2}^{0} \rightarrow \tilde{\ell}_{2}^{ \pm} \tau^{\mp}$ & 0 & 0.00001 \\
$\tilde{\ell}_{3} \rightarrow \tilde{\chi}_{1}^{0} e$ & 1 & 0.62 & $\tilde{\chi}_{2}^{0} \rightarrow \tilde{\ell}_{3}^{ \pm} e^{\mp}$ & 0.019 & 0.0117 \\
$\tilde{\ell}_{3} \rightarrow \tilde{\chi}_{1}^{0} \mu$ & 0 & 0.335 & $\tilde{\chi}_{2}^{0} \rightarrow \tilde{\ell}_{3}^{ \pm} \mu^{\mp}$ & 0 & 0.0069 \\
$\tilde{\ell}_{3} \rightarrow \tilde{\chi}_{1}^{0} \tau$ & 0 & 0.044 & $\tilde{\chi}_{2}^{0} \rightarrow \tilde{\ell}_{3}^{ \pm} \tau^{\mp}$ & 0 & 0.0234 \\
& & $\tilde{\chi}_{2}^{0} \rightarrow \tilde{\nu} \nu$ & 0.401 & 0.401 \\
\end{tabular}

Table 1: Branching ratios of $\tilde{\ell}_{1,2,3}$ and $\tilde{\chi}_{2}^{0}$ decays in the LFC and LFV cases for the SPS1a' scenario. The LFV case is characterized by the following LFV parameters: $M_{E, 12}^{2}=30 \mathrm{GeV}^{2}, M_{E, 13}^{2}=850 \mathrm{GeV}^{2}$ and $M_{E, 23}^{2}=600 \mathrm{GeV}^{2}$, for which one has $\left(m_{\tilde{\ell}_{1}}, m_{\tilde{\ell}_{2}}, m_{\tilde{\ell}_{3}}\right)=(106.4,125.1,126.2) \mathrm{GeV}$. In the LFC case (i.e. in the case where $\left.M_{E, 12}^{2}=M_{E, 13}^{2}=M_{E, 23}^{2}=0\right)$ one has $\left(m_{\tilde{\ell}_{1}}, m_{\tilde{\ell}_{2}}, m_{\tilde{\ell}_{3}}\right)=(107.4,125.2,125.3) \mathrm{GeV}$. Note that $\left(\tilde{\ell}_{1}, \tilde{\ell}_{2}, \tilde{\ell}_{3}\right)=\left(\tilde{\tau}_{1}, \tilde{\mu}_{1}, \tilde{e}_{1}\right)$ in the LFC case and that $\left(\tilde{\ell}_{1}, \tilde{\ell}_{2}, \tilde{\ell}_{3}\right) \sim\left(\tilde{\tau}_{R}, \tilde{\mu}_{R}, \tilde{e}_{R}\right)$ in the LFV case.

structures in the $e \tau$ and $\mu \tau$ invariant mass distributions, should be clearly visible on top of the background. Note that the usual method for background suppression, by taking the sum $N\left(e^{+} e^{-}\right)+N\left(\mu^{+} \mu^{-}\right)-N\left(e^{ \pm} \mu^{\mp}\right)$, is not applicable in the case of LFV searches. Instead one has to study the individual pair mass spectra. Nevertheless, one can expect that these peaks will be well observable [21. Here note also that the tau lepton could be identified by a hadronic "tau jet" though the double-edge structures in the $e \tau$ and $\mu \tau$ spectra would get a smearing effect due to a neutrino emission [13]. To show more clearly the observability of such LFV signals a detailed Monte Carlo study would be necessary. This, however, is beyond the scope of the present paper. 
(a) $100 \Gamma_{\text {tot }}^{-1} d \Gamma\left(\tilde{\chi}_{2}^{0} \rightarrow \ell_{i}^{ \pm} \ell_{j}^{\mp} \tilde{\chi}_{1}^{0}\right) / d m\left(\ell_{i}^{ \pm} \ell_{j}^{\mp}\right)\left[\mathrm{GeV}^{-1}\right]$

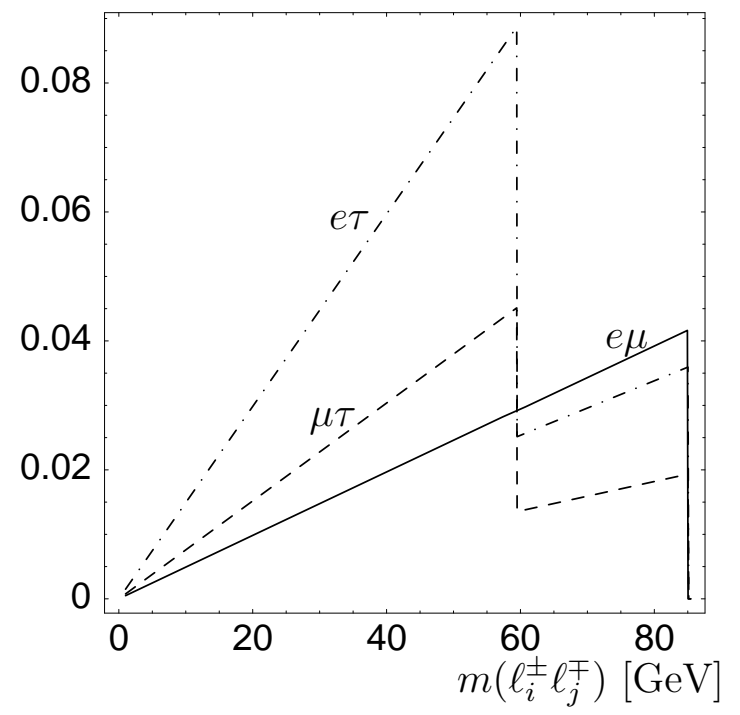

(b) $100 \Gamma_{\text {tot }}^{-1} d \Gamma\left(\tilde{\chi}_{2}^{0} \rightarrow \ell^{+} \ell^{-} \tilde{\chi}_{1}^{0}\right) / d m\left(\ell^{+} \ell^{-}\right)\left[\mathrm{GeV}^{-1}\right]$

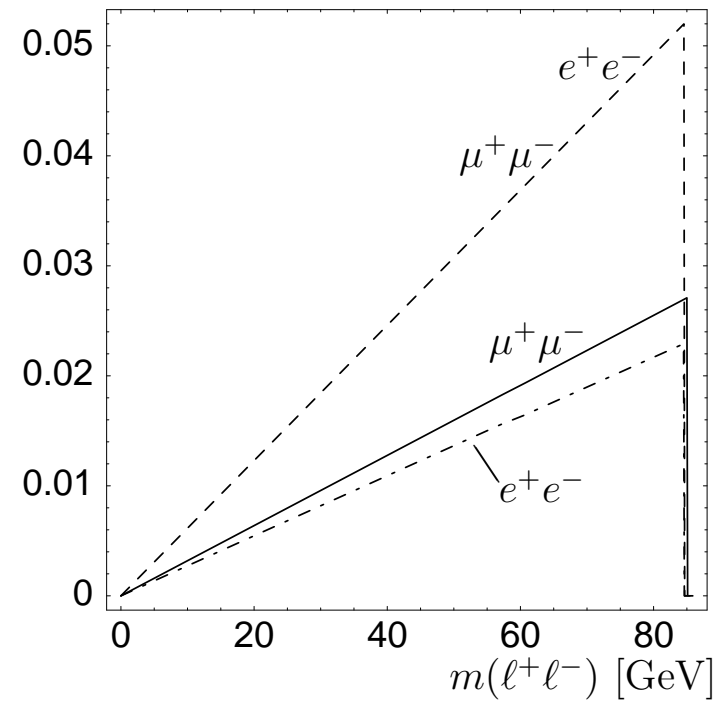

Figure 4: Invariant mass spectra $100 \Gamma_{\text {tot }}^{-1} d \Gamma\left(\tilde{\chi}_{2}^{0} \rightarrow \ell_{i} \ell_{j} \tilde{\chi}_{1}^{0}\right) / d m\left(\ell_{i} \ell_{j}\right)$ versus $m\left(\ell_{i} \ell_{j}\right)$. In (a) we show the "flavour violating" spectra summed over charges in the LFV case for the SPS1a' scenario: $e^{ \pm} \mu^{\mp}$ (full line), $e^{ \pm} \tau^{\mp}$ (dashed dotted line) and $\mu^{ \pm} \tau^{\mp}$ (dashed line) and in (b) we show the "flavour conserving" spectra: $e^{+} e^{-}$(dashed line) and $\mu^{+} \mu^{-}$(dashed line) are for the LFC case in the SPS1a' scenario, and $e^{+} e^{-}$(dashed dotted line) and $\mu^{+} \mu^{-}$(full line) are for the LFV case in the SPS1a' scenario.

\section{Summary}

To summarize, we have studied the effect of SUSY lepton flavour violation on the decay chains $\tilde{\chi}_{2}^{0} \rightarrow \ell_{i}^{\mp} \tilde{\ell}_{j}^{ \pm} \rightarrow \ell_{i}^{\mp} \ell_{k}^{ \pm} \tilde{\chi}_{1}^{0}$, which may arise from cascade decays of gluinos and squarks at LHC. As an example, we have adopted the SPS1a' scenario supplemented with lepton flavour violating entries in the soft SUSY breaking mass matrix $M_{E}^{2}$, with two and three generation mixings in the right slepton sector which give the most important contributions to the LFV decays. Additional mixings in the left-left and/or left-right sectors do not lead to a significant change of the LFV signals.

We have found that the most recent experimental bounds on flavour violating lepton decays allow for sizable flavour violating $\tilde{\chi}_{2}^{0}$ decay branching ratios with the following upper limits: $\operatorname{BR}\left(\tilde{\chi}_{2}^{0} \rightarrow e \mu \tilde{\chi}_{1}^{0}\right) \lesssim 2 \%, \operatorname{BR}\left(\tilde{\chi}_{2}^{0} \rightarrow e \tau \tilde{\chi}_{1}^{0}\right) \lesssim 4 \%$ and $\operatorname{BR}\left(\tilde{\chi}_{2}^{0} \rightarrow \mu \tau \tilde{\chi}_{1}^{0}\right) \lesssim 3 \%$. Moreover, a strong correlation between the branching ratios $\operatorname{BR}\left(\tilde{\chi}_{2}^{0} \rightarrow e \tau \tilde{\chi}_{1}^{0}\right)\left(\operatorname{BR}\left(\tilde{\chi}_{2}^{0} \rightarrow \mu \tau \tilde{\chi}_{1}^{0}\right)\right)$ and $\operatorname{BR}\left(\tau^{-} \rightarrow e^{-} \gamma\right)\left(\operatorname{BR}\left(\tau^{-} \rightarrow \mu^{-} \gamma\right)\right)$ 
is found. This would imply that if $\mathrm{BR}\left(\tau^{-} \rightarrow e^{-} \gamma\right)$ or $\mathrm{BR}\left(\tau^{-} \rightarrow \mu^{-} \gamma\right)$ were measured not to be much below the current upper bound, then the signals of the corresponding lepton flavour violating neutralino decays should also be accessible at future collider experiments. Furthermore, a sizable $\operatorname{BR}\left(\tilde{\chi}_{2}^{0} \rightarrow e \mu \tilde{\chi}_{1}^{0}\right)$ can be compatible with a small $\operatorname{BR}\left(\mu^{-} \rightarrow e^{-} \gamma\right)$ in case of three generation mixing differently from two generation mixing cases as previously studied.

In particular, we have studied the impact of LFV due to three slepton generation mixing on the di-lepton mass distributions from the decays $\tilde{\chi}_{2}^{0} \rightarrow \tilde{\ell} \ell^{\prime} \rightarrow \ell^{\prime} \ell^{\prime \prime} \tilde{\chi}_{1}^{0}$ measured at LHC. For the di-lepton spectra of two leptons with equal flavour we have found that LFV leads to a reduction of the height of the end-point peaks. This reduction is different for $e^{+} e^{-}, \mu^{+} \mu^{-}$and $\tau^{+} \tau^{-}$channels. This means, for example, that even in case of nearly degenerate masses of $\tilde{e}_{R}$ and $\tilde{\mu}_{R}$ the $e^{+} e^{-}$mass spectrum can be significantly different from that of $\mu^{+} \mu^{-}$due to three slepton generation mixing. For two leptons of different flavours we have found that novel and characteristic edge structures in the distributions, such as a double-edge structure in the $e \tau$ and $\mu \tau$ mass spectra can appear. The double-egde structure stems from the mass difference between $\tilde{\tau}_{1}\left(\sim \tilde{\tau}_{R}\right)$ and $\tilde{e}_{R}, \tilde{\mu}_{R}$. The appearance of such remarkable structures provides a powerful test of SUSY lepton flavour violation at LHC and useful informations on the flavour structure of the slepton sector can be obtained. In such a case the additional

peak may allow for a more precise measurement of the mass of $\tilde{\ell}_{1}\left(\sim \tilde{\tau}_{1}\right)$. Finally, we have also worked out the conditions for the appearance of such a double-edge structure in the di-lepton mass distributions for scenarios different from the SPS1a' scenario.

\section{Acknowledgments}

We thank I. Hinchliffe for useful discussions. This work is supported by the 'Fonds zur Förderung der wissenschaftlichen Forschung' (FWF) of Austria, project No. P16592N02 and by Acciones Integradas Hispano-Austriaca. W.P. is supported by a MCyT Ramon y Cajal contract, by the Spanish grant BFM2002-00345, by the European Commission Human Potential Program RTN network HPRN-CT-2000-00148 and partly by the Swiss 'Nationalfonds'. 


\section{References}

[1] M. L. Brooks et al. [MEGA Collaboration], Phys. Rev. Lett. 83, (1999) 1521 arXiv:hep-ex/9905013.

[2] U. Bellgardt et al. [SINDRUM Collaboration], Nucl. Phys. B 299, (1988) 1.

[3] B. Aubert et al. [BABAR Collaboration], arXiv:hep-ex/0508012.

[4] B. Aubert et al. [BABAR Collaboration], arXiv:hep-ex/0502032.

[5] Y. Yusa et al. [Belle Collaboration], Phys. Lett. B 589, (2004) 103 arXiv:hep-ex/0403039.

[6] F. Riepenhausen, in Proceedings of the Sixth Conference on the Intersections of Particle and Nuclear Physics, T. W. Donnelly, ed. (AIP, New York, 1997), p. 34.

[7] M. Maltoni, T. Schwetz, M. A. Tortola and J. W. F. Valle, New J. Phys. 6, (2004) 122 arXiv:hep-ph/0405172.

[8] J. Hisano, T. Moroi, K. Tobe and M. Yamaguchi, Phys. Rev. D 53, (1996) 2442.

[9] J. F. Donoghue, H. P. Nilles and D. Wyler, Phys. Lett. B 128 (1983) 55; L. J. Hall, V. A. Kostelecky and S. Raby, Nucl. Phys. B 267 (1986) 415; F. Borzumati and A. Masiero, Phys. Rev. Lett. 57, (1986) 961; F. Gabbiani and A. Masiero, Phys. Lett. B 209 (1988) 289; J. S. Hagelin, S. Kelley and T. Tanaka, Nucl. Phys. B 415 (1994) 293; R. Barbieri and L. J. Hall, Phys. Lett. B 338 (1994) 212; F. Gabbiani, E. Gabrielli, A. Masiero and L. Silvestrini, Nucl. Phys. B 477 (1996) 321; J. Hisano, T. Moroi, K. Tobe and M. Yamaguchi, Phys. Lett. B 391, (1997) 341 [Erratum-ibid. B 397, (1997) 357]; J. Hisano, D. Nomura and T. Yanagida, Phys. Lett. B 437, (1998) 351.

[10] N. V. Krasnikov, Phys. Lett. B 388, (1996) 783; M. Nowakowski and A. Pilaftsis, Nucl. Phys. B 461, (1996) 19 arXiv:hep-ph/9508271; J. Hisano, M. M. Nojiri, Y. Shimizu and M. Tanaka, Phys. Rev. D 60, (1999) 055008; D. Nomura, Phys. Rev. D 64, (2001) 075001; M. Guchait, J. Kalinowski and P. Roy, Eur. Phys. J. C 21, (2001) 163; W. Porod and W. Majerotto, Phys. Rev. D 66 (2002) 015003 arXiv:hep-ph/0201284; D. F. Carvalho, J. R. Ellis, M. E. Gomez, S. Lola and J. C. Romao, Phys. Lett. B 618, (2005) 162 arXiv:hep-ph/0206148; 
D. A. Demir, Phys. Lett. B 571, (2003) 193 arXiv:hep-ph/0303249; W. Porod, arXiv:hep-ph/0410318, F. Deppisch, H. Päs, A. Redelbach, R. Rückl and Y. Shimizu, Phys. Rev. D 69 (2004) 054014 arXiv:hep-ph/0310053; K. Hamaguchi and A. Ibarra, JHEP 0502 (2005) 028 arXiv:hep-ph/0412229; N. Oshimo, Eur. Phys. J. C 39 (2005) 383 arXiv:hep-ph/0409018; P. Paradisi, arXiv:hep-ph/0505046.

[11] N. Arkani-Hamed, H. C. Cheng, J. L. Feng and L. J. Hall, Phys. Rev. Lett. 77, (1996) 1937; Nucl. Phys. B 505, (1997) 3.

[12] K. Agashe and M. Graesser, Phys. Rev. D $61 \quad$ (2000) 075008 arXiv:hep-ph/9904422.

[13] I. Hinchliffe and F. E. Paige, Phys. Rev. D 63, (2001) 115006.

[14] J. Hisano, R. Kitano and M. M. Nojiri, Phys. Rev. D 65 (2002) 116002.

[15] A. Dedes, J. R. Ellis and M. Raidal, Phys. Lett. B 549, (2002) 159 arXiv:hep-ph/0209207.

[16] D. J. H. Chung, L. L. Everett, G. L. Kane, S. F. King, J. Lykken and L. T. Wang, Phys. Rept. 407 (2005) 1 arXiv:hep-ph/0312378.

[17] http://spa.desy.de/spa

[18] F. E. Paige, eConf C960625, SUP114 (1996) arXiv:hep-ph/9609373.

[19] H. Bachacou, I. Hinchliffe and F. E. Paige, Phys. Rev. D 62, (2000) 015009 arXiv:hep-ph/9907518.

[20] B. C. Allanach, C. G. Lester, M. A. Parker and B. R. Webber, JHEP 0009 (2000) 004 arXiv:hep-ph/0007009.

[21] I. Hinchliffe, private communication. 